\title{
An in vitro investigation into the role of bone marrow-derived mesenchymal stem cells in the control of disc degeneration
}

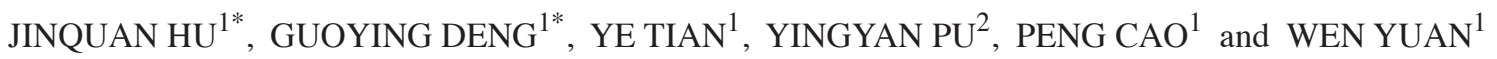 \\ ${ }^{1}$ Department of Orthopedic Surgery, Changzheng Hospital, Shanghai 200023; \\ ${ }^{2}$ Institute of Neuroscience and Key Laboratory of Molecular Neurobiology of The Ministry of Education, \\ Neuroscience Research Center of Changzheng Hospital, Second Military Medical University, Shanghai 200433, P.R. China
}

Received October 11, 2014; Accepted June 30, 2015

DOI: $10.3892 / \mathrm{mmr} .2015 .4139$

\begin{abstract}
Excessive apoptosis and high expression levels of interleukin-1 $\beta$ (IL-1 $\beta$ ) in disc cells have been reported to serve important roles in intervertebral disc degeneration (IVDD). Previous studies investigating mesenchymal stem cells (MSCs) have indicated potential for their use in the treatment of IVDD. However, the therapeutic potential and anti-apoptotic ability of MSCs remains to be fully elucidated. The present study aimed to establish an in vitro model for bone marrow-derived MSC (BMSC) therapy by investigating the anti-apoptotic effects, in addition to the migration of BMSCs to nucleus pulposus (NP) cells stimulated by IL-1 $\beta$. A co-culture system of BMSCs and NP cells was founded. Following inflammatory stimulation, the NP cells exhibited increased indexes for inflammation-induced degeneration. The degenerative and apoptotic indexes were significantly reduced when NP cells were co-cultured with BMSCs. Compared with the indirect co-culture group, the direct co-culture group exhibited an improved capacity for anti-apoptosis. In addition, IL-1 $\beta$-stimulated NP cells attracted and mediated the migration of BMSCs. Mitochondrial transfer from BMSCs to NP cells by tunneling nanotubes was also observed. In conclusion, the anti-apoptosis and the migration, in addition to mitochondrial transfer associated with BMSC treatments in IVDD, were investigated in vitro in the present study. The interaction between stimulated NP cells and BMSCs is likely involved in to simulating the in vivo process of stem cell-mediated repair.
\end{abstract}

Correspondence to: Dr Peng Cao or Dr Wen Yuan, Department of Orthopedic Surgery, Changzheng Hospital, 415 Fengyang Road, Shanghai 200023, P.R. China

E-mail: mdpinocao@163.com

E-mail: wenyuan2014@126.com

*Contributed equally

Key words: mesenchymal stem cells, disc degeneration, apoptosis, co-culture system, migration, tunneling nanotubes

\section{Introduction}

Diseases resulting from the degeneration of the intervertebral discs have become increasing threats to quality of life due to a rapidly aging society (1). The abnormal apoptosis or age-associated apoptosis of nucleus pulposus (NP) cells is suggested to serve a key role in the development of degenerative disc diseases (2-4). High levels of interleukin-1 $\beta$ (IL-1 $\beta$ ) are present in degenerating intervertebral discs and there is a positive response of intervertebral disc cells to IL-1 $\beta$ inhibition $(5,6)$. This indicates that the inflammatory process, in particular the IL-1 $\beta$-induced degradation of proteoglycans and type II collagen, results in damage to and accelerates the apoptosis of NP cells and annulus fibrosus cells, thereby leading to the development of degenerative disc diseases (5,7-10). Reducing inflammation and the deleterious impact of apoptosis is therefore critical for the treatment of degenerative disc diseases.

Due to their numerous advantages, including their abundance, high activity, low immunogenicity, marked proliferation, differentiation potential and nutrient secretion, mesenchymal stem cells (MSCs) have been widely used in general transplantation research, with positive effects (11-13). MSC transplantation, which exhibits beneficial effects in the treatment of degenerative disc diseases, including the inhibition of intervertebral disc degeneration (IVDD), has been reported to increase the number of cells in intervertebral discs and results in the partial recovery of intervertebral disc height (14-16). However, the specific effects and mechanisms of MSCs on NP cells remain to be fully elucidated. Although certain previous studies have used green fluorescent protein (GFP)-transfected cells as a means of distinguishing between cell types, it remains challenging to accurately separate transfected cells when assessing intercellular interactions (17). The complication of in vivo studies is whether the observed therapeutic effect arises from in situ cells being 'nourished' by BMSCs $(12,18,19)$, or is instead an artifact of BMSCs, which exhibit high activity and differentiation potential (13). In vivo studies are therefore, inherently limited. In order to further investigate the mechanisms underlying MSC therapy at the cellular level, the present study used a Transwell assay involving non-contacting and contacting co-culture systems to simulate the in vivo paracrine interactions between cells and directed migration $(20,21)$. Unlike previous studies, the 
anti-apoptotic and migratory capabilities, in addition to mitochondrial transfer through tunneling nanotube (TnT) formation of BMSCs were directly assessed in vitro. The present study was able to measure specific alterations in NP cells via paracrine mechanisms and mitochondrial transfer resulting from MSCs.

\section{Materials and methods}

Ethics statement. The male Sprague-Dawley rats (age, 3 months; weight, 250-300 g) used in the current study were provided by the Second Military Medical University Laboratory Animal Center (Shanghai, China). The rats were housed under a $12 \mathrm{~h}$ light/dark cycle at constant temperature $\left(25^{\circ} \mathrm{C}\right)$ and humidity, with ad libitum access to food and water. All experiments were approved by the Animal Ethical Committee of the Second Military Medical University (no. 13071002114).

Isolation and culture of BMSCs and NP cells from Sprague-Dawley rats. Primary BMSCs were isolated and cultured, as described previously (16). The harvested cells were centrifuged at $500 \mathrm{xg}$ for $10 \mathrm{~min}$ at $4^{\circ} \mathrm{C}$ and then resuspended in complete Dulbecco's modified Eagle's medium (DMEM)/F-12 with $10 \%$ fetal bovine serum (FBS), $100 \mu \mathrm{g} / \mathrm{ml}$ streptomycin and $100 \mathrm{U} / \mathrm{ml}$ penicillin (all purchased from Gibco Life Technologies, Carlsbad, CA, USA). Cells at passage 3 were subjected to flow cytometric analysis (Cytomics FC500; Beckman Coulter, Brea, CA, USA) to assess the expression of the surface markers, cluster of differentiation 29 (CD29), CD90, CD31 and CD45. The osteogenic and adipogenic differentiation potentials of the cells were also assessed by Alizarin red staining (Beyotime Institute of Biotechnology, Haimen, China) and Oil Red $\mathrm{O}$ staining (Beyotime Institute of Biotechnology).

The rat NP cells were isolated, as described previously (22). The NP cells were seeded into $60 \mathrm{~mm}$ tissue culture dishes and grown in complete culture medium at $37^{\circ} \mathrm{C}$ with $5 \% \mathrm{CO}_{2}$. The culture medium was replaced every 2-3 days. The cells were passaged by digestion with $0.25 \%$ trypsin (Gibco Life Technologies) once they reached $80-90 \%$ confluence. The NP cells at passage 3 in culture medium were used for subsequent experiments.

Establishment of a co-culture system and cell treatment. In order to construct the co-culture system, NP cells were plated at a constant density of $2 \times 10^{4}$ cells $/ \mathrm{cm}^{2}$ into certain culture plates and were subsequently divided into different groups based on specific treatments. Two steps were included and each lasted $24 \mathrm{~h}$. In the first step, serum-free medium with or without IL-1 $\beta$ (PeproTech, Inc., Rocky Hill, NJ, USA) was used for stimulation and subsequently, complete medium with or without BMSCs (the ratio was 1:2 to the NP cells) was used for co-culture intervention. The groups used were as follows: i) Control group, the cells were treated with serum-free medium, which was then replaced with complete medium; ii) No-culture group, the cells were subjected to intervention treatment with IL-1 $\beta$ (20 ng/ml), which was then replaced with complete medium; iii) Indirect co-culture group, following stimulation with IL-1 $\beta(20 \mathrm{ng} / \mathrm{ml})$, the BMSCs were co-cultured using a Transwell (pore size $0.4 \mu \mathrm{m}$; EMD
Millipore, Billerica, MA, USA) in complete medium; iv) direct co-culture group, following stimulation with IL-1 $\beta(20 \mathrm{ng} / \mathrm{ml})$, an identical quanitity of GFP BMSCs were directly added to the NP cells with complete medium.

Reverse transcription-quantitative polymerase chain reaction ( $R T$ - $q P C R)$. The total RNA was isolated from the NP cells using TRIzol reagent (Invitrogen Life Technologies, Carlsbad, CA, USA) and the RNA concentration was measured photometrically (NanoDrop 2000/2000c; Thermo Fisher Scientific, Inc., Wilmington, DE, USA). Synthesis of the cDNA was performed using the RevertAid First Strand cDNA Synthesis kit (Thermo Fisher Scientific, Waltham, MA, USA), according to the manufacturer's instructions. PCR analysis was performed using gene-specific primers (Table I) for GAPDH, a disintegrin and metalloproteinase with thrombospondin motifs 4 (ADAMTS-4), ADAMTS-5, matrix metalloproteinase 13 (MMP-13), tissue inhibitor of metalloproteinases 1 (TIMP-1) and caspase-3 (Sangon Biotech Co., Ltd., Shanghai, China). RT-qPCR was performed using a MyiQ ${ }^{\mathrm{TM}}$ Detection System (Bio-Rad Laboratories, Inc., Hercules, CA, USA) with SYBR Green Real-time PCR Master mix (Toyobo Co., Ltd., Osaka, Japan). Amplification of the target cDNA was normalized against the expression of GAPDH. The relative levels of the target mRNA expression were calculated using the $2^{-\Delta \Delta \mathrm{Ct}}$ method (23).

Terminal deoxynucleotide transferase dUTP nick end labeling (TUNEL) apoptosis analysis. Following fixation in freshly prepared $4 \%$ paraformaldehyde (Roche Diagnostics, Basel, Switzerland) for $1 \mathrm{~h}$ at $4^{\circ} \mathrm{C}$, the cells were incubated with $3 \% \mathrm{H}_{2} \mathrm{O}_{2}$ and $0.1 \%$ Triton $\mathrm{X}-100$ for $10 \mathrm{~min}$ and washed three times with phosphate-buffered saline (PBS) at each step. The cells were stained using the In Situ Cell Death Detection kit (Roche Diagnostics) and counterstained with Hoechst 33258 (Beyotime Institute of Biotechnology), according to the manufacturer's instructions. Apoptotic alterations were measured by fluorescence microscopy (BX51; Olympus, Tokyo, Japan).

Caspase-3 activity assay. Caspase-3 activity was determined using a Caspase-3 Activity kit (Beyotime Institute of Biotechnology), which is based on the caspase-3-mediated conversion of acetyl-Asp-Glu-Val-Asp p-nitroanilide into the yellow formazan product, $\mathrm{p}$-nitroaniline, according to the manufacturer's instructions. The activity of caspase-3 was quantified on a microplate spectrophotometer (Biotek Instruments, Inc., Winooski, VT, USA) at 405 nm. Caspase-3 activity was expressed as the fold-change in enzyme activity compared with that of synchronized cells.

Detection of apoptotic incidence by flow cytometry. Apoptotic incidence was detected using the Annexin V-Fluorescein Isothiocyanate (FITC) [Phycoerythrin (PE) for direct co-culture]/propidium iodide (PI) Apoptosis Detection kit I (BD Pharmingen, San Diego, CA, USA), according to the manufacturer's instructions. The samples were analyzed on a fluorescence activated cell sorter (Cytomics FC500; Beckman Coulter) within $1 \mathrm{~h}$. Apoptotic cells, including annexin-positive/PI-negative in addition to double-positive 

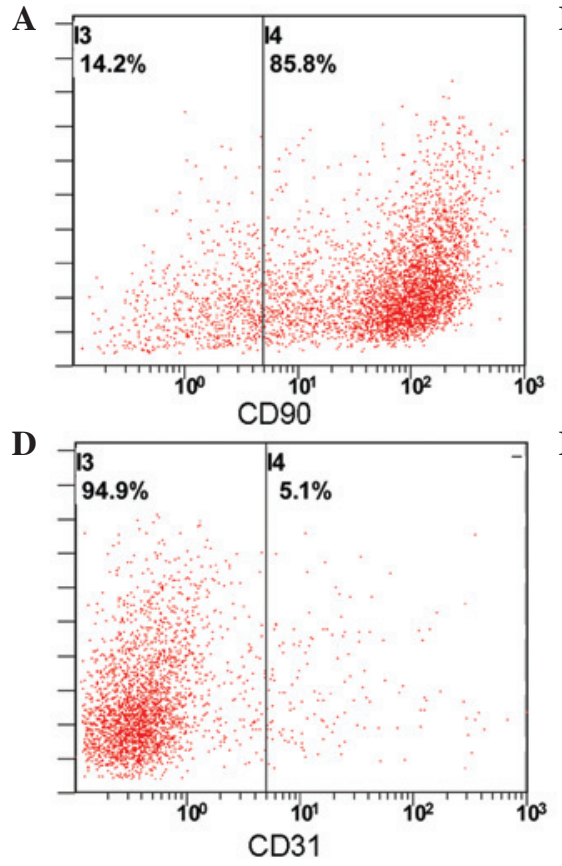

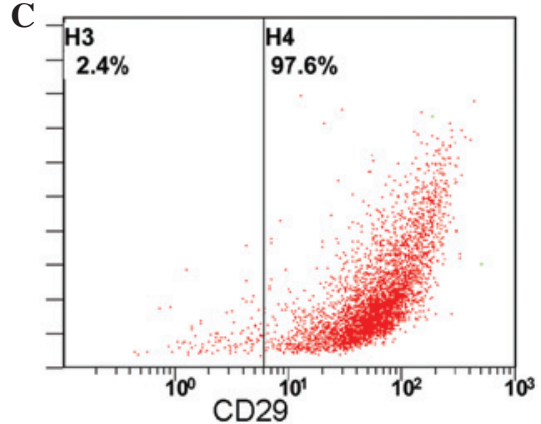

$\mathbf{F}$

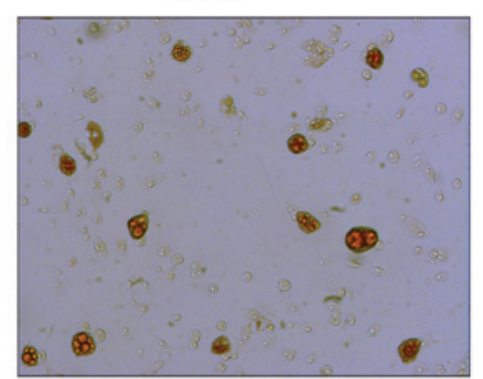

Figure 1. Identification of isolated BMSCs. Flow cytometric analysis of (A) CD90, (B) CD45, (C) CD29 and (D) CD31. BMSCs (>85\%) were positive for CD29 and CD90, whereas $<0.1 \%$ of the BMSCs were positive for CD31 and CD45. Following 2 week induced differentiation, (E) osteogenic differentiation of the cells was examined by Alizarin red staining and $(\mathrm{F})$ adipogenic differentiation was assessed by Oil Red O staining. BMSCs, bone marrow-derived mesenchymal stem cells; CD90, cluster of differentiation 90.

cells, were counted and represented as a percentage of the total cell count.

Detection of migration of BMSCs. The migratory ability of BMSCs was assessed using Transwell plates (Corning Inc., Corning, NY, USA), which were $6.5 \mathrm{~mm}$ in diameter with $8 \mu \mathrm{m}$ pore filters. The BMSCs were harvested and equal numbers of cells were added to each group of Transwell plates. Following incubation for $24 \mathrm{~h}$ at $37^{\circ} \mathrm{C}$, the cells that had not migrated from the upper side of the filter were scraped off with a cotton swab and the filters were stained with Coomassie Blue (Beyotime Institute of Biotechnology). The number of cells that had migrated to the lower side of the filter were counted under a light microscope at a magnification of $\mathrm{x} 400$ in five randomly selected fields.

Staining cells and confocal microscopy. To trace intercellular exchange of mitochondria, GFP BMSCs were separately labeled with MitoTracker ${ }^{\circledR}$ Deep Red (Invitrogen Life Technologies), according to the manufacturer's instructions. Briefly, the cells were resuspended in pre-warmed $\left(37^{\circ} \mathrm{C}\right)$ staining solution, containing the MitoTracker ${ }^{\circledR}$ probe (50 nM) for $30 \mathrm{~min}$ in complete medium. Following staining, the cells were washed three times in PBS and resuspended in fresh pre-warmed medium and were directly added into IL-1 $\beta$ stimulated NP cells on glass slides for $24 \mathrm{~h}$. Following the addition of the cells, the glass slides with two types of cells were washed with PBS and were labeled with DAPI (10 $\mu \mathrm{g} / \mathrm{ml}, 5 \mathrm{~min}$; Beyotime Institute of Biotechnology). Images were captured by confocal microscopy (TCS SP5; Leica Microsystems GmbH, Wetzlar, Germany) for $1 \mathrm{~h}$ and were subsequently analyzed by Leica LAS AF Lite_2.5.0_7266 software.
Statistical analysis. All statistical analyses were performed in triplicate. All data are presented as the mean \pm standard error. Differences between the groups were analyzed by one way analysis of variance $\left({ }^{*} \mathrm{P}<0.05,{ }^{* *} \mathrm{P}<0.01\right)$ using GraphPad Prism software, version 5.0 (GraphPad Software, Inc., La Jolla, CA, USA).

\section{Results}

Identification of isolated BMSCs. Flow cytometric analysis was used to detect CD90 (Fig. 1A), CD45 (Fig. 1B), CD29 (Fig. 1C) and CD90 (Fig. 1D) expression. BMSCs (>85\%) were positive for CD31 and CD45, whereas $<0.1 \%$ of BMSCs were positive for CD31 and CD45. Following 2 weeks of induced differentiation, osteogenic differentiation was examined by Alizarin red staining (Fig. 1E), and adipogenic differentiation was assessed by Oil Red O staining (Fig. 1F). The results suggest that cells cultured in this manner can be induced to differntiate into both osteogenic and adipogenic cells.

Co-culture of BMSCs with IL-1 $\beta$-stimulated NP cells reduces degenerative enzyme gene expression. Compared with the unstimulated NP cells, the NP cells in the no co-culture groups exhibited markedly higher expression levels of ADAMTS-4, ADAMTS-5, caspase-3 and MMP-13 following inflammatory stimulation (Fig. 2A-D), whereas the expression of TIMP-1 was reduced (Fig. 2E). Compared with the no co-culture group, various indexes were significantly reduced in the co-culture group. In addition, the expression of TIMP-1 was increased.

Co-culture of BMSCs with IL-1 $\beta$-stimulated NP cells reduces the number of TUNEL-stained cells. TUNEL staining allows 
Table I. Primer sequences used for reverse transcription-quantitative polymerase chain reaction.

\begin{tabular}{lll}
\hline Gene & Primer name & Sequence (5'-3') \\
\hline GAPDH & GAPDH-F & CCATCAACGACCCCTTCATT \\
ADAMTS-4 & GAPDH-R & ATTCTCAGCCTTGACTGTGC \\
ADAMTS-5 & ADAMTS-F & ACAATGGCTATGGACACTGCCTCT \\
& ADAMTS-R & TGTGGACAATGGCTTGAGTCAGGA \\
MMP-13 & ADAMTS-F & GTCCAAATGCACTTCAGCCACGAT \\
& ADAMTS-R & AATGTCAAGTTGCACTGCTGGGTG \\
TIMP-1 & MMP-13-F & CCCTGGAGCCCTGATGTTT \\
Caspase-3 & MMP-13-R & CTCTGGTGTTTGGGGTGCT \\
& TIMP-F & ATAGTGCTGGCTGTGGGGTGTG \\
& TIMP-R & TGATCGCTCTGGTAGCCCTTCTC \\
\hline
\end{tabular}

ADAMTS, a disintegrin and metalloproteinase with thrombospondin motifs; MMP, matrix metalloproteinase; TIMP-1, tissue inhibitor metalloproteinase 1; F, forward; R, reverse.

A

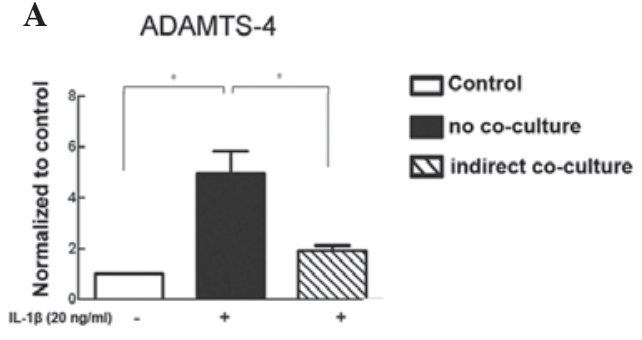

D

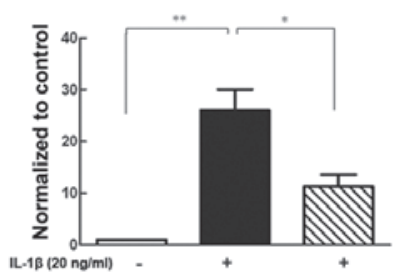

B

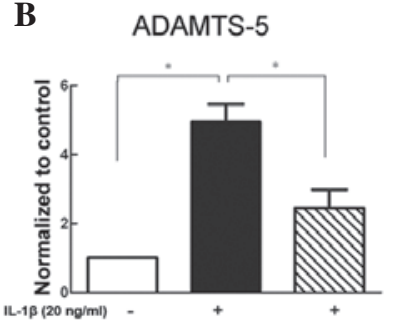

C

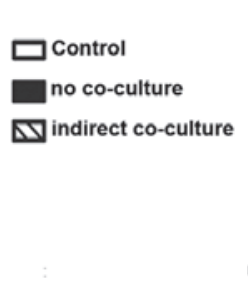

E TIMP-1
C Caspase-3

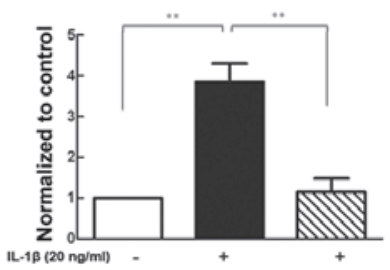

Figure 2. Reverse transcription-quantitative polymerase chain reaction analysis of the expression of (A) ADAMTS-4, (B) ADAMTS-5, (C) caspase-3, (D) MMP-13 and (E) TIMP-1. Significant reductions were observed following IL-1 $\beta$ (20 ng/ml) stimulation, and there was a clear increase in expression subsequent to bone marrow-derived mesenchymal stem cell indirect co-culture. The data are presented as the mean \pm standard error of three independent experiments $\left({ }^{*} \mathrm{P}<0.05\right.$ and $\left.{ }^{* *} \mathrm{P}<0.01\right)$. ADAMTS, a disintegrin and metalloproteinase with thrombospondin motifs; MMP, matrix metalloproteinase; TIMP, tissue inhibitor of metalloproteinase; IL-1 $\beta$; interleukin-1 $\beta$.

for the detection and quantification of apoptosis at the cellular level. The number of TUNEL-positive cells increased with inflammatory factors $(20 \mathrm{ng} / \mathrm{ml})$, whereas the number of TUNEL-positive cells was reduced in the co-culture group (Fig. 3A).

Co-culture of BMSCs with IL-1 $\beta$-stimulated NP cells reduces the activity of caspase-3. The results of the caspase- 3 activity assay demonstrated that compared with the normal group, the caspase- 3 activities in the 20 and $50 \mathrm{ng} / \mathrm{ml}$ inflammatory factor treatment groups were significantly increased. In each indirect co-culture group, the caspase-3 activity was marginally lower compared with the no co-culture group (Fig. 3B).
Co-culture of BMSCs with IL-1 $\beta$-stimulated NP cells reduces the apoptotic incidence. Flow cytometric analysis of annexin V-FITC PE/PI staining demonstrated that the level of apoptosis was significantly increased in the NP cells stimulated with inflammatory factors. In addition, the apoptotic rate increased in line with the increase in inflammatory factor concentration. These results indicated that co-culturing NP cells with BMSCs significantly reduced the apoptotic rate of different levels of IL-1 $\beta$. This suggested that direct co-culture may have improved the anti-apoptotic effects compared with the indirect co-culture (Fig. 4A and B). Notably, the apoptotic rate of the added BMSCs in the direct co-culture group was markedly low (Fig. 4C). 
A
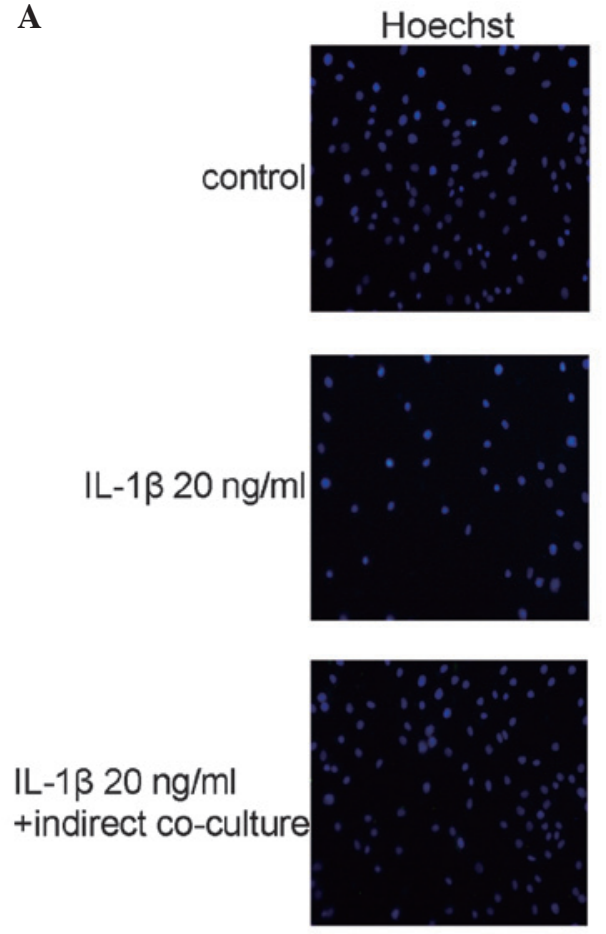

TUNEL
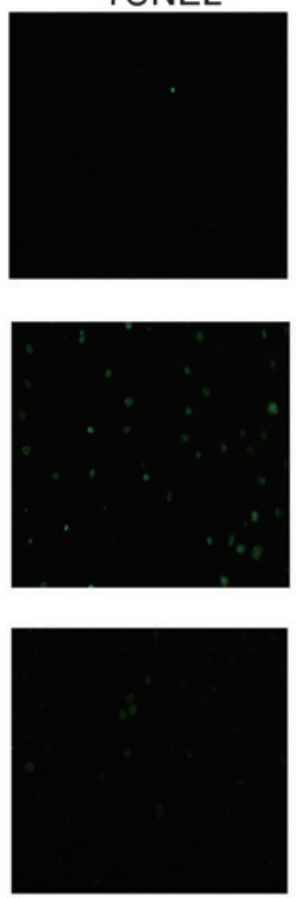

B

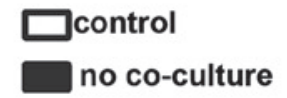

\section{$\square$ indirect co-culture}

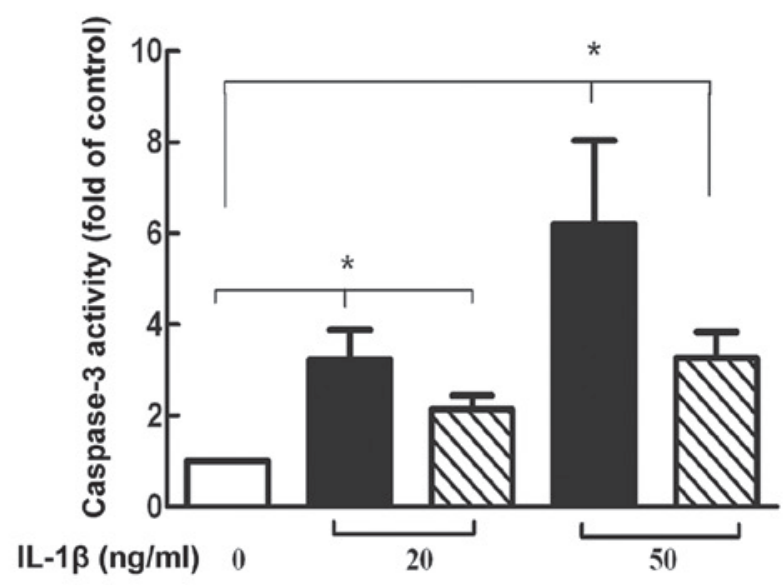

Figure 3. Apoptosis rate detection by TUNEL-staining and caspase-3 ELISA kit. (A) The apoptotic response of the nucleus pulposus cells without simulation was low. At $24 \mathrm{~h}$ following stimulation with IL-1 $(20 \mathrm{ng} / \mathrm{ml})$, the level of apoptosis was significantly increased and the indirect co-culture significantly reversed this increase. (B) Using the caspase-3 ELISA kit, comparable results were obtained with 20 and $50 \mathrm{ng} / \mathrm{ml}$ IL-1 $\beta$ ("P<0.05). IL-1 $\beta$; interleukin-1 $\beta$.

A
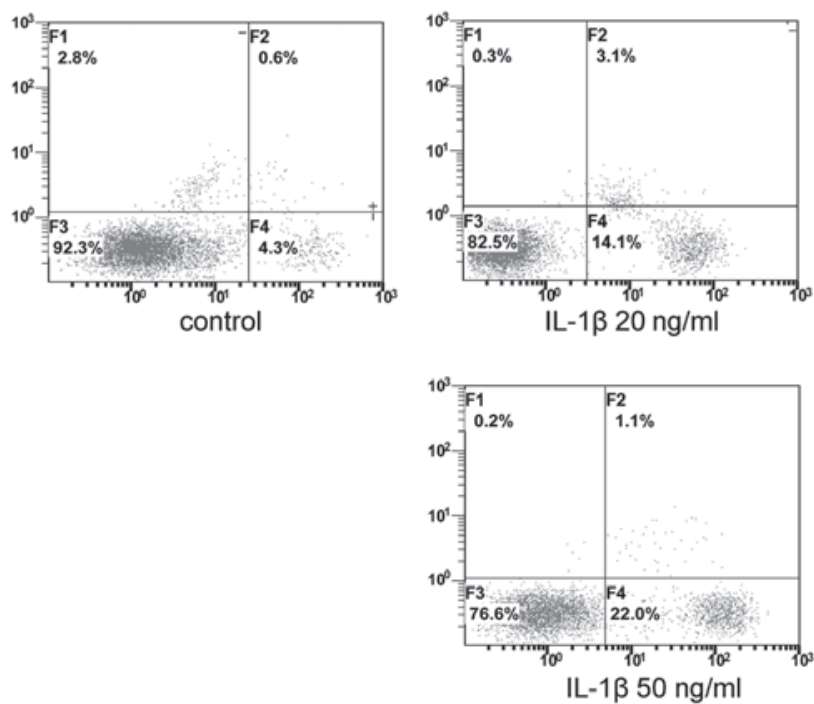

B

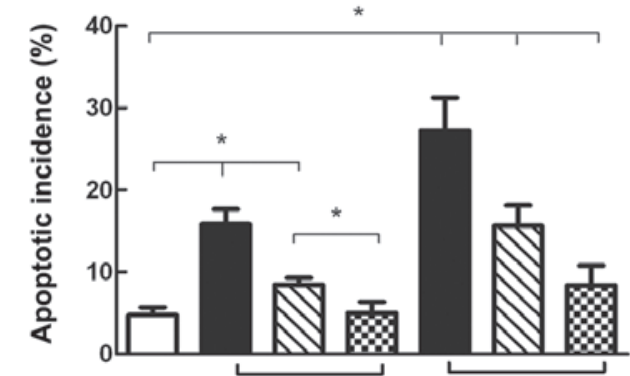

$\mathrm{IL}-1 \beta(\mathrm{ng} / \mathrm{ml}) \quad 0$
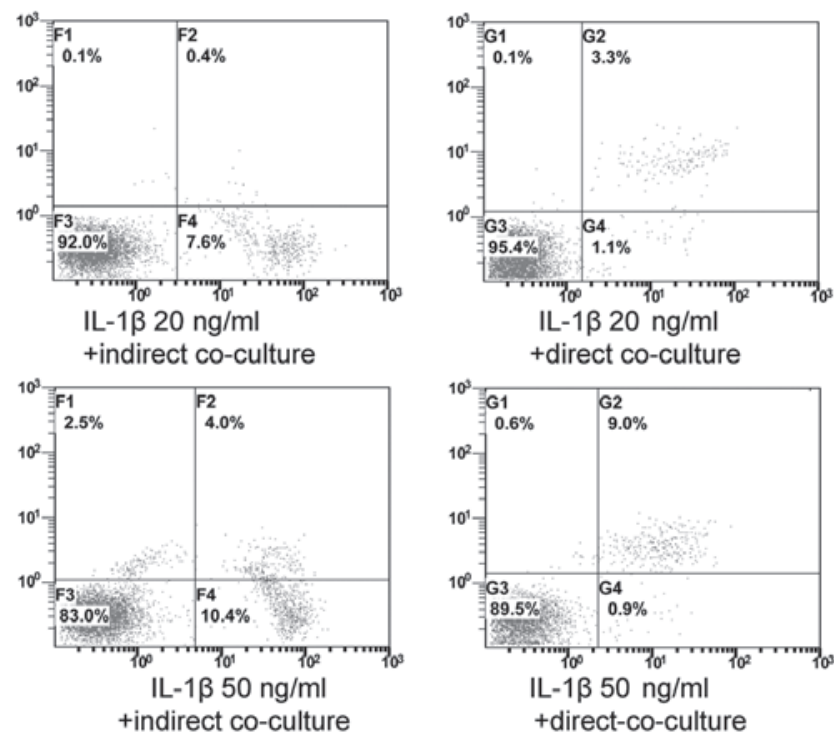

C

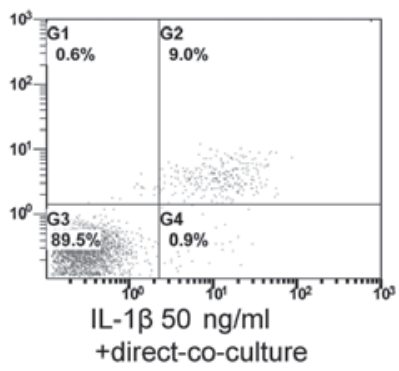

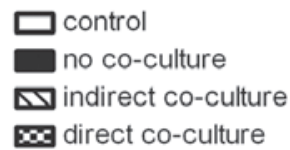

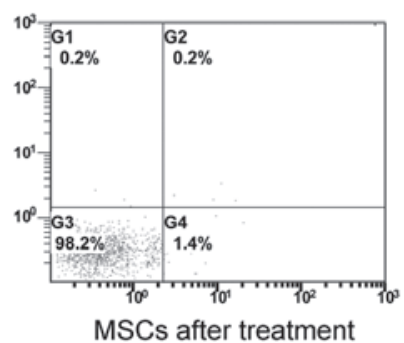

Figure 4. Evaluation of apoptotic incidence. (A) Following double staining with annexin V-fluorescein isothiocyanate (phycoerythrin for direct co-culture) and propidium iodide, $0 \mathrm{ng} / \mathrm{ml}$ resulted in a normal apoptosis rate of the NP cells. The apoptotic rates of the NP cells were increased following different levels of IL-1 $\beta$ stimulation, then were reduced when indirectly co-cultured with BMSCs, with a more marked reduction observed in the direct co-culture. (B) The apoptotic incidences of the NP cells in different groups were compared ( $\left.{ }^{*} \mathrm{P}<0.05\right)$. (C) The directly co-cultured BMSCs were minimally impacted. NP, nucleus pulposus; IL-1 $\beta$, interleukin-1 $\beta$; BMSCs, bone marrow-derived mesenchymal stem cells. 
A

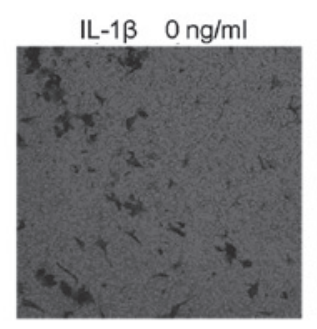

$20 \mathrm{ng} / \mathrm{ml}$

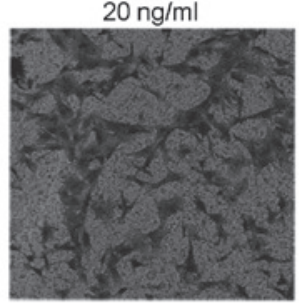

$50 \mathrm{ng} / \mathrm{ml}$

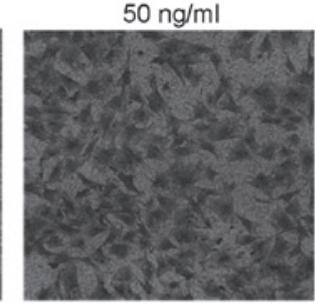

B

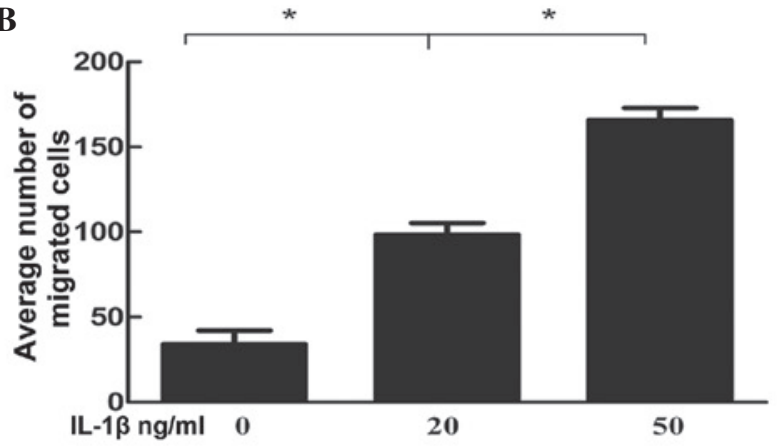

Figure 5. BMSC migration assay with a Transwell chamber. (A) The number of cells that migrated through the pores were counted in 10 random high power fields for each group. (B) The average number of migrated cells for each group were compared. Cell damage resulting from IL-1 $\beta$ stimulation enhanced the migratory ability of BMSCs in a dose-dependent manner ("P<0.05). BMSCs, bone marrow-derived mesenchymal stem cells; IL-1 $\beta$; interleukin-1 $\beta$.

A

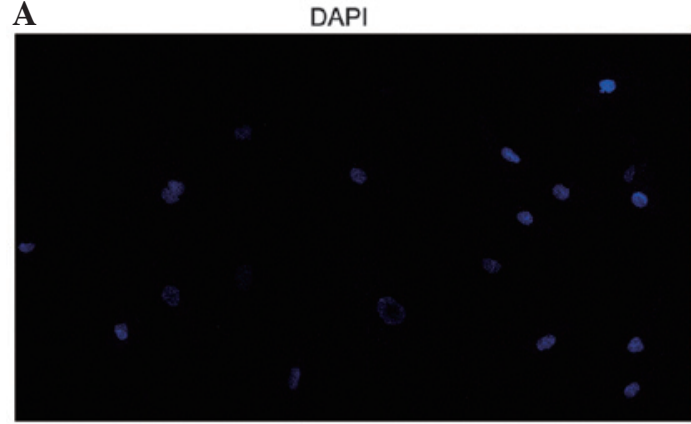

C

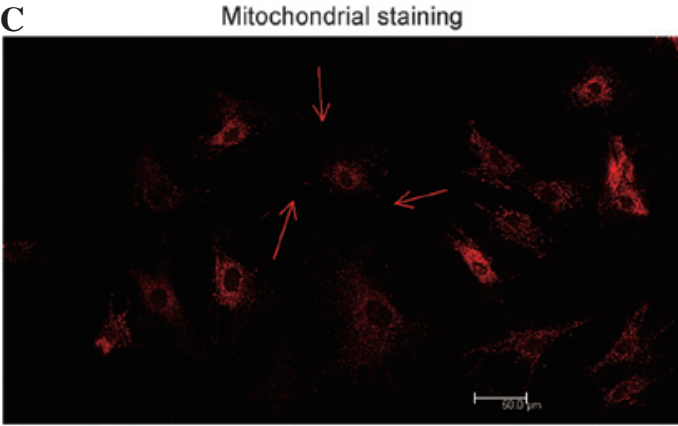

B

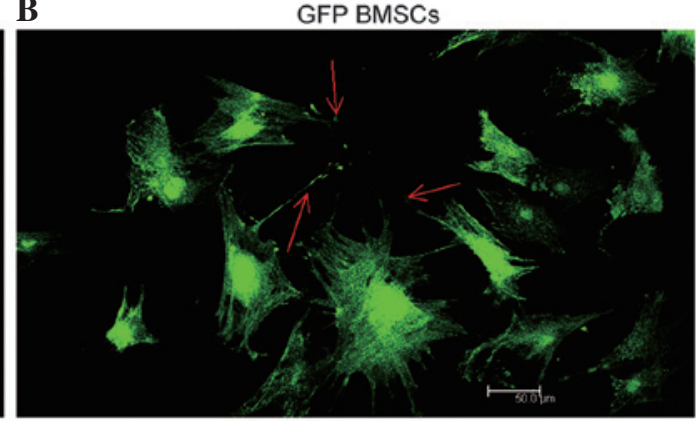

D

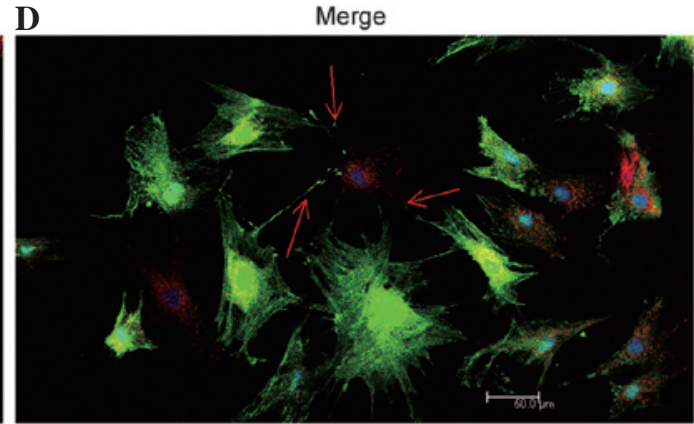

Figure 6. Mitochondrial transfer measured by confocal microscopy. Phase-contrast images were captured in three fluorescence channels. (A) DAPI, (B) GFP (fluorescein isothiocyanate), (C) MitoTracker ${ }^{\circledR}$ Deep Red and (D) merged image. The arrows indicate thin TnT-like structures. Pre-lableling MitoTracker ${ }^{\circledR}$ (red) mitochondria were observed transferring from BMSCs to stimulated nucleus pulposus cells through TnT-like structures at $24 \mathrm{~h}$ of co-culture. Scale bars, $50 \mu \mathrm{m}$. TnT, tunneling nanotubes; GFP, green fluorescent protein; BMSCs, bone marrow-derived mesenchymal stem cells.

$I L-1 \beta$ stimulation of NP cells increases the migratory ability of the BMSCs. The number of cells, which migrated through the pores were counted under 10 random high power fields for each group (Fig. 5A), which were then compared (Fig. 5B). Amongst all the groups, the differences between the negative control and IL-1 $\beta$ pre-induced groups were statistically significant. Cell damage resulting from IL-1 $\beta$ enhanced the migratory ability of BMSCs in a dose-dependent manner.

Mitochondrial transfer occurs between direct co-cultured NP cells and BMSCs. The arrows in Fig. 6 indicate that TnT-like structures were observed between BMSCs and NP cells by confocal microscopy. Mitochondria were clearly observed in the red fluorescence channel, transferring from relabeled GFP BMSCs to stimulated NP cells via TnTs (Fig. 6).

\section{Discussion}

In the present study, a novel model of BMSC intervention was established in IVDD and the anti-apoptotic ability of BMSCs in stimulated NP cells was observed to act via a paracrine mechanism. In addition, the migration driven by IL- $1 \beta$ stimulation and directed mitochondrial transfer was observed.

IL-1 $\beta$ has been used to induce inflammatory responses in NP cells $(6,9,24)$. Following stimulation with IL-1 $\beta$ for $24 \mathrm{~h}$, the majority of degeneration indexes were increased and apoptosis was induced. To simulate in vivo MSC-mediated damage repair processes following inflammatory stimulation, Transwell chambers were used to physically separate the two cell types. The use of a Transwell chamber with a $0.4 \mu \mathrm{m}$ pore size ensured that only secreted factors were easily passed through the pores, while preventing BMSCs from migrating 
through the pores via amoeboid-like formations (20). Previous in vivo studies have reported that these intercellular interactions involve the indirect effects of cytokines, in addition to the influence of cell migration and direct cell to cell contacts $(25,26)$. Through a series of experiments, the present study successfully simulated and confirmed the directional migration of BMSCs toward the inflammatory factor-stimulated cells. However, the model for BMSC migration failed to completely mimic MSC action in vivo and was only suitable to separately investigate the effects of BMSCs on damaged cells.

In addition to paracrine effects and migration, direct cell to cell communication must be addressed. The observation of TnTs between MSCs and other cell types has been reported by numerous previous studies $(27,28)$. MSCs are capable of transferring mitochondria to cells with severely compromised mitochondrial function via TnTs (29). In the present study, only GFP BMSCs were pre-labeled with MitoTracker ${ }^{\circledR}$ Red following $24 \mathrm{~h}$ direct co-culture, however, the pre-simulated NP cells were labeled red. Due to the fact that mitochondrial transfer by TnTs was commonly observed in the present study between GFP BMSCs and NP cells, which had suffered cellular damage (identified by DAPI), it was suggested that migration of BMSCs may be directed and BMSCs may transfer mitochondria into cells with severe damage. Unfortunately, quantifying this is challenging and further investigation is required.

The co-culture model established in the present study demonstrated that BMSCs exert beneficial anti-apoptotic effects on inflammatory factor-stimulated NP cells via paracrine mechanisms. The anti-inflammatory and anti-apoptotic capacities of MSCs, in addition to their mechanisms of action, have been reported in other fields. MSCs co-cultured in inflammatory medium have been reported to inhibit the activation of nuclear factor $\kappa-\mathrm{B}$ and thereby control a series of inflammatory reactions (30). It has been reported that MSCs secrete factors, including transforming growth factor- $\beta$ and prostaglandin E2, which can inhibit the activation of lymphocytes, thereby also contributing to the suppression of inflammation (31-33). The paracrine-mediated anti-apoptotic effects are likely to be based on the activity of growth factors produced by MSCs, which are able to prevent oxidative stress by increasing the activity of antioxidants and normalizing mitochondrial function (34). Previous studies have demonstrated with in vivo and in vitro models that protein kinase B (Akt) was significantly increased in cells treated with MSCs and MSC culture medium, and that Akt exerts an anti-apoptotic effect through the phosphorylation of the BLC-associated cell death promoter protein $(12,35,36)$. In addition, serum may function as a positive factor, whcih reduces the overall inflammatory and apoptotic indexes (9). Significant differences were detected between the co-culture experiments with BMSCs and the monoculture experiments lacking BMSCs. Through paracrine mechanisms, BMSCs were suggested to exert an anti-apoptotic effect on the damaged cells, migrating to the damaged cells and communicating with them via TnTs. Therefore, in models of IVDD, MSC transplantation has been reported to exert a significant therapeutic effect $(15,17)$.

In conclusion, BMSCs actively migrate towards inflammatory NP cells and effectively reduce the apoptotic rate in NP cells following inflammatory stimulation via paracrine effects and mitochondrial transfer through direct TnTs connections. The in vitro model established in the present study simulates, to a certain extent, these paracrine anti-apoptotic effects, in addition to the migratory behavior of BMSCs. This model provided a simple and accurate means to investigate the mechanisms underlying the potentially beneficial therapeutic effects of BMSCs.

\section{Acknowledgements}

The present study was supported by the Shanghai Science and Technology Committee Project of International Cooperation (grant no. 13430721000) and the Joint Research Project on Major Diseases of Shanghai Health System (grant no. 2013ZYJB0502).

\section{References}

1. Waddell G: Low back pain: A twentieth century health care enigma. Spine (Phila Pa 1976) 21: 2820-2825, 1996

2. Ariga K, Miyamoto S, Nakase T, Okuda S, Meng W, Yonenobu K and Yoshikawa $\mathrm{H}$ : The relationship between apoptosis of endplate chondrocytes and aging and degeneration of the intervertebral disc. Spine (Phila Pa 1976) 26: 2414-2420, 2001.

3. Lotz JC, Colliou OK, Chin JR, Duncan NA and Liebenberg E: Compression-induced degeneration of the intervertebral disc: An in vivo mouse model and finite-element study. Spine (Phila Pa 1976) 23: 2493-2506, 1998.

4. Yamada K, Sudo H, Iwasaki K, Sasaki N, Higashi H, Kameda Y, Ito M, Takahata M, Abumi K, Minami A, et al: Caspase 3 silencing inhibits biomechanical overload-induced intervertebral disk degeneration. Am J Pathol 184: 753-764, 2014.

5. Le Maitre CL, Freemont AJ and Hoyland JA: The role of interleukin-1 in the pathogenesis of human intervertebral disc degeneration. Arthritis Res Ther 7: R732-R745, 2005.

6. Tian Y, Yuan W, Fujita N, Wang J, Wang H, Shapiro IM and Risbud MV: Inflammatory cytokines associated with degenerative disc disease control aggrecanase-1 (ADAMTS-4) expression in nucleus pulposus cells through MAPK and NF- $\kappa B$. Am J Pathol 182: 2310-2321, 2013.

7. Roberts S, Caterson B, Menage J, Evans EH, Jaffray DC and Eisenstein SM: Matrix metalloproteinases and aggrecanase: Their role in disorders of the human intervertebral disc. Spine (Phila Pa 1976) 25: 3005-3013, 2000.

8. Zhang CC, Zhou JS, Hu JG, Wang X, Zhou XS, Sun BA, Shao C and Lin Q: Effects of IGF-1 on IL-1 $\beta$-induced apoptosis in rabbit nucleus pulposus cells in vitro. Mol Med Rep 7: 441-444, 2013.

9. Zhao CQ, Liu D, Li H, Jiang LS and Dai LY: Interleukin-1beta enhances the effect of serum deprivation on rat annular cell apoptosis. Apoptosis 12: 2155-2161, 2007.

10. Takahashi H, Suguro T, Okazima Y, Motegi M, Okada Y and Kakiuchi T: Inflammatory cytokines in the herniated disc of the lumbar spine. Spine (Phila Pa 1976) 21: 218-224, 1996.

11. Pittenger MF, Mackay AM, Beck SC, Jaiswal RK, Douglas R, Mosca JD, Moorman MA, Simonetti DW, Craig S and Marshak DR: Multilineage potential of adult human mesenchymal stem cells. Science 284: 143-147, 1999.

12. Morigi M, Rota C, Montemurro T, Montelatici E, Lo Cicero V, Imberti B, Abbate M, Zoja C, Cassis P, Longaretti L, et al: Life-sparing effect of human cord blood-mesenchymal stem cells in experimental acute kidney injury. Stem Cells 28: 513-522, 2010.

13. Richardson SM, Walker RV, Parker S, Rhodes NP, Hunt JA, Freemont AJ and Hoyland JA: Intervertebral disc cell-mediated mesenchymal stem cell differentiation. Stem Cells 24: 707-716, 2006.

14. Sakai D, Mochida J, Yamamoto Y, Nomura T, Okuma M, Nishimura K, Nakai T, Ando K and Hotta T: Transplantation of mesenchymal stem cells embedded in Atelocollagen gel to the intervertebral disc: A potential therapeutic model for disc degeneration. Biomaterials 24: 3531-3541, 2003.

15. Hiyama A, Mochida J, Iwashina T, Omi H, Watanabe T, Serigano K, Tamura F and Sakai D: Transplantation of mesenchymal stem cells in a canine disc degeneration model. J Orthop Res 26: 589-600, 2008. 
16. Crevensten G, Walsh AJ, Ananthakrishnan D, Page P, Wahba GM, Lotz JC and Berven S: Intervertebral disc cell therapy for regeneration: Mesenchymal stem cell implantation in rat intervertebral discs. Ann Biomed Eng 32: 430-434, 2004.

17. Sakai D, Mochida J, Iwashina T, Watanabe T, Nakai T, Ando K and Hotta T: Differentiation of mesenchymal stem cells transplanted to a rabbit degenerative disc model: Potential and limitations for stem cell therapy in disc regeneration. Spine (Phila $\mathrm{Pa}$ 1976) 30: 2379-2387, 2005.

18. Strassburg S, Richardson SM, Freemont AJ and Hoyland JA: Co-culture induces mesenchymal stem cell differentiation and modulation of the degenerate human nucleus pulposus cell phenotype. Regen Med 5: 701-711, 2010.

19. Bi B, Schmitt R, Israilova M, Nishio H and Cantley LG: Stroma cells protect against acute tubular injury via an endocrine effect. J Am Soc Nephrol 18: 2486-2496, 2007.

20. Yang SH, Wu CC, Shih TT, Sun YH and Lin FH: In vitro study on interaction between human nucleus pulposus cells and mesenchymal stem cells through paracrine stimulation. Spine (Phila Pa 1976) 33: 1951-1957, 2008.

21. Li X, Zhang Y, Yeung SC, Liang Y, Liang X, Ding Y, Ip MS, Tse HF, Mak JC and Lian Q: Mitochondrial transfer of induced pluripotent stem cells-derived mesenchymal stem cells to airway epithelial cells attenuates cigarette Smoke-induced damage. Am J Respir Cell Mol Biol 51: 455-465, 2014.

22. Risbud MV, Guttapalli A, Stokes DG, Hawkins D, Danielson KG, Schaer TP, Albert TJ and Shapiro IM: Nucleus pulposus cells express HIF-1 alpha under normoxic culture conditions: A metabolic adaptation to the intervertebral disc microenvironment. J Cell Biochem 98: 152-159, 2006.

23. Karaliotas GI, Mavridis K, Scorilas A and Babis GC: Quantitative analysis of the mRNA expression levels of BCL2 and BAX genes in human osteoarthritis and normal articular cartilage: An investigation into their differential expression. Mol Med Rep 12: 4514-4521, 2015

24. Smith LJ, Chiaro JA, Nerurkar NL, Cortes DH, Horava SD, Hebela NM, Mauck RL, Dodge GR and Elliott DM: Nucleus pulposus cells synthesize a functional extracellular matrix and respond to inflammatory cytokine challenge following long-term agarose culture. Eur Cell Mater 22: 291-301, 2011.

25. Wang Z, Wang Y, Gutkind JS, Wang Z, Wang F, Lu J, Niu G, Teng $\mathrm{G}$ and Chen $\mathrm{X}$ : Engineered mesenchymal stem cells with enhanced tropism and paracrine secretion of cytokines and growth factors to treat traumatic brain injury. Stem Cells 32: 456-467, 2015
26. Kawai T, Katagiri W, Osugi M, Sugimura Y, Hibi H and Ueda M: Secretomes from bone marrow-derived mesencyhmal stromal cells enhance periodontal tissue regeneration. Cytotherapy 17: 369-381, 2015.

27. Figeac F, Lesault PF, Le Coz O, Damy T, Souktani R, Trébeau C, Schmitt A, Ribot J, Mounier R, Guguin A, et al: Nanotubular crosstalk with distressed cardiomyocytes stimulates the paracrine repair function of mesenchymal stem cells. Stem Cells 32: 216-230, 2014

28. Vallabhaneni KC, Haller H and Dumler I: Vascular smooth muscle cells initiate proliferation of mesenchymal stem cells by mitochondrial transfer via tunneling nanotubes. Stem Cells Dev 21: 3104-3113, 2012

29. Gerdes HH, Bukoreshtliev NV and Barroso JF: Tunneling nanotubes: A new route for the exchange of components between animal cells. FEBS Lett 581: 2194-2201, 2007.

30. Yagi H, Soto-Gutierrez A, Navarro-Alvarez N, Nahmias Y, Goldwasser Y, Kitagawa Y, Tilles AW, Tompkins RG, Parekkadan B and Yarmush Ml: Reactive bone marrow stromal cells attenuate systemic inflammation via sTNFR1. Mol Ther 18: 1857-1864, 2010.

31. Aggarwal S and Pittenger MF: Human mesenchymal stem cells modulate allogeneic immune cell responses. Blood 105: 1815-1822, 2005.

32. Groh ME, Maitra B, Szekely E and Koç ON: Human mesenchymal stem cells require monocyte-mediated activation to suppress alloreactive T cells. Exp Hematol 33: 928-934, 2005.

33. Ulivi V, Tasso R, Cancedda R and Descalzi F: Mesenchymal stem cell paracrine activity is modulated by platelet lysate: Induction of an inflammatory response and secretion of factors maintaining macrophages in a proinflammatory phenotype. Stem Cells Dev 23: 1858-1869, 2014.

34. García-Fernández M, Delgado G, Puche JE, González-Barón S and Castilla Cortázar I: Low doses of insulin-like growth factor I improve insulin resistance, lipid metabolism and oxidative damage in aging rats. Endocrinology 149: 2433-2442, 2008

35. Eliopoulos N, Zhao J, Bouchentouf M, Forner K, Birman E, Yuan S, Boivin MN and Martineau D: Human marrow-derived mesenchymal stromal cells decrease cisplatin renotoxicity in vitro and in vivo and enhance survival of mice post-intraperitoneal injection. Am J Physiol Renal Physiol 299: F1288-F1298, 2010.

36. Kuwana H, Terada Y, Kobayashi T, Okado T, Penninger JM, Irie-Sasaki J,Sasaki T and Sasaki S: The phosphoinositide-3 kinase gamma-Akt pathway mediates renal tubular injury in cisplatin nephrotoxicity. Kidney Int 73: 430-445, 2008. 